\title{
Court-circuit dans les Transformateurs à 3 enroulements
}

\author{
Par MM. Barkère, Ingénieur aux Etablissements Schneider \\ Chef des Travaux Electrotechniques à l'Ecole Centrale des Arts al Manufactures \\ et R. Lschapasse, Ancien élive de l'Ecole Polylechnique.
}

Comme suile à létude qui a paru dernièrement dans cette mème revue, sur des transformateurs de grande puissance à trois enroulements, nous allons voir l'influence du troisième enroulement sur la valeur du courant de court-circuit dans les diffẻrents cas possibles.

10 Impédance de court-circuit. - Supposons d'abord le cas, qui se produira au début de l'exploitation du réseau considéré, où le transformateur sera alimenté seulement par son primaire. le secondaire débitant sa charge sur le réseau secondaire à 45.600 $V$., mais ce dernier ne comportant pas de station génératrice, le troisième enroulement est supposé à vide, nalimentant pas le condensateur synchrone.

Dans ces conditions, un court-circuit sur l'un des deux cnrou-. lements secondaires n'est pas influencé par l'autre, mais si un court-circuit se produit à la fois sur les deux secondaires, l'impédance effective du transformateur pour limiter le courant de court-circuit est modifiée. Pour étudier son influence, considérons la figure 1 et les deux enroulements $\mathrm{A}$ et $\mathrm{B}$ réunis en

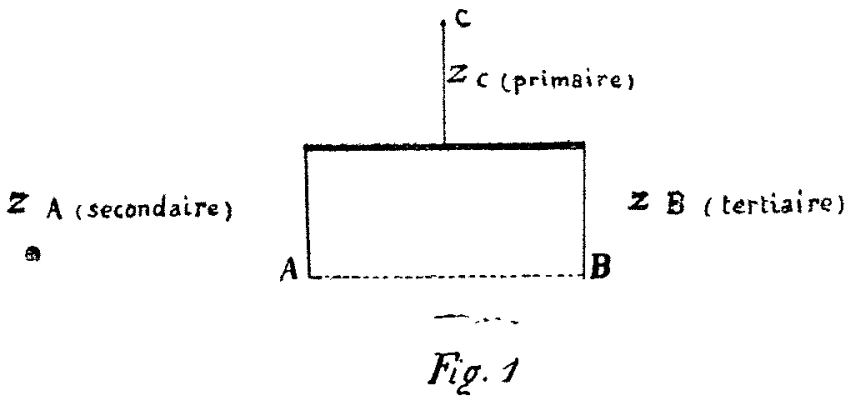

parallèle (cas équivalent à celui d'un court-circuit simultané sur ces deux enroulements).

Déterminons l'impédance effective du système $\mathrm{AB}, \mathrm{C}$, on aura :

$$
Z=Z_{\mathrm{ab}}+Z_{\mathrm{c}}=\frac{1}{\frac{1}{Z_{\mathrm{a}}}+\frac{1}{Z_{\mathrm{b}}}}+Z_{\mathrm{c} \tilde{z}}
$$

Etant donné la très faible valeur des résistances par rapport aux réactances (5\% environ) nous ne considérerons que lés réactances; nous aurons done:

$$
\begin{aligned}
& X_{a}=\frac{X_{a b}+X_{a c}-X_{b c}}{2} \\
& X_{b}=\frac{X_{a b}+X_{b c}-X_{a c}}{2}
\end{aligned}
$$

et (1) donne :

$$
\mathrm{x}=\frac{\mathrm{X}_{\mathrm{a}}+\mathrm{X}_{\mathrm{b}}}{\mathrm{X}_{\mathrm{ab}}}+\mathrm{X}_{\mathrm{c}}=\frac{\mathrm{X}_{\mathrm{a}} \mathrm{X}_{\mathrm{b}}+\mathrm{X}_{\mathrm{c}} \mathrm{X}_{\mathrm{ab}}}{\mathrm{X}_{\mathrm{ab}}}
$$

et en remplaçant $X_{a}$ et $X_{b}$ par leurs valeurs écrites plus haul ct en tenant compte de ce que :

$\mathrm{X}_{\mathrm{bc}}+\mathrm{X}_{\mathrm{ac}}-\mathrm{X}_{\mathrm{ab}}=2 \mathrm{X}_{\mathrm{c}}$, nous en concluons :

$\frac{X_{a c} X_{b c}-X_{c}^{2}}{X_{0}}$ (réactance effective du système $A, B, C$, nous avons vu que l'on avail:

$$
\begin{array}{rlrl}
\mathrm{X}_{\mathrm{ac}}=11,5 \% & \mathrm{X}_{\mathrm{a}}=9,675 \% \\
\mathrm{X}_{\mathrm{bc}}=8,85 \% & \mathrm{X}_{\mathrm{b}}=6,97 \% \\
\mathrm{X}_{\mathrm{ab}}=16,6 \% & \mathrm{X}_{\mathrm{c}}=1,87 \% \\
\mathrm{X}=\frac{11,5 \times \frac{8,85-1,87}{106}}{106}=5,9 \%
\end{array}
$$

le courant de court-circuit serait donc: $\frac{100}{5,9}=16,95$ fois le courant normal.

Mais ceci se produirait dans le cas théorique ou l'alimentation d'énergie se ferait directement au primaire, c'est-à-dire sans autre réactance dans le circuit d'alimentation que celle du transformateur étudié ; or, dans le cas qui nous intéresse la réactance jusquau transformateur (somme des réactances des alternateurs, des transformateurs de départ el de la ligne) est de $40,5 \%$.

Il en résulte :

$\mathrm{X}$ totale $=46,4 \%$ soil un courant de court-circuil de : $\frac{100}{46,4}=2,16$ fois le courant normal.

Cherchant la répartition de ce courant dans le secondaire $A$ et dans le tertiaire $\mathrm{B}$ on a :

$$
\frac{\mathrm{KVA}_{\mathrm{a}}}{\mathrm{KVA} \text { totaux }}=\frac{\mathrm{Z}_{\mathrm{b}}}{\mathrm{Z}_{\mathrm{ab}}} \quad \frac{\mathrm{KV} \Lambda_{\mathrm{b}}}{\mathrm{KVA} \text { totaux }}=\frac{\mathrm{Z}_{\mathrm{b}}}{\mathrm{Z}_{\mathrm{ab}}}
$$

soit :

$$
\begin{aligned}
& \mathrm{KV \Lambda}_{\mathrm{a}}=\frac{6,97}{16,6} \times 2,16=0,907 \text { fois le courant normal. } \\
& \mathrm{KVA}_{\mathrm{b}}=\frac{9.675}{16,6} \times 2,16=1,255 \text { fois le courant normal. }
\end{aligned}
$$

soit pour $3.000 \mathrm{KVA}$ normaux par transformateur monophasé :

$$
\begin{aligned}
& \text { au primaire }(\mathrm{C}): 2,16 \times 3.000=6.480 \mathrm{KVA} \\
& \text { au secondaire }(\Lambda): 0,907 \times 3.000=2.720 \mathrm{KV} \Lambda \\
& \text { au terliaire }(\mathrm{B}): 1,255 \times 3.000=3.770 \mathrm{KV} \Lambda
\end{aligned}
$$

On voit que ces courants sont relativement très faibles.

Cela est dû à la réactance assez considérable du circuit primaire.

Remarquons que les alternateurs sont munis de régulateurs automatiques qui tendent à augmenter l'excitation lors d'un court-circuit dans le réseau.

Cependant comme ils sont munis également de limitateurs d'intensité, ces deux actions tendent à se neutraliser, du moins pour le fonctionnement du disjoncteur destiné à couper le transformateur.

Nous pouvons donc conserver la réactance de $40,5 \%$ introduite plus haut dans le calcul.

$2^{\circ}$ Court-circuit monophasé. - Il peut se produire soil entre

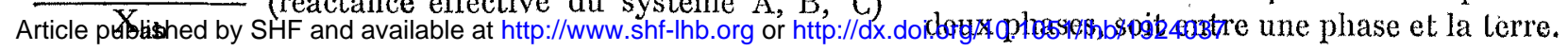


Rappelons ici 4 théoremes indiqués par M. Boyajian, dans sa théorie des transformaleurs à 3 enroulements.

Thorcime I. - - Une charge monophasce (mais non sur le neuLre) sur une ligne triphasee, egale au liers de la capacite en IrVA du systeme triphasé, produit une chute inductive en $\%$ egale aux $2 / 3$ de la chule inductive en $\%$ du système pour sa capacité.

Thiorème II. - L'impédance en \% d'un appareil triphasé (par exemple d'un groupe de transformateurs) pour une charge monopluséce entre phase et neutre doit être évaluće dans chaque cas particulier; cependant, du côté primaire la charge monophasée est transportéc par les conducteurs, et l'impédance du systeme comnceté aux conducteurs primaires suit le théorème I.

Si les neutres de plusicurs appareils sont réunis ensemble (par exemple si le neutre du réseau est mis à la terre en un certain nombre de points) ces appareils devraient être considéés comme étant en parallèle pour une charge conducteur-neutre on pour tel court-circuit.

Theorime III. .... Une charge conducteur-neutre se distribue de manière a rencontrer dans son trajet la chute inductive la plus petite possible. Quant cette charge peut se distribuer sans magnétiser le noyau du transformateur c'est ainsi qu'elle le fait.

Thiorène $I V$. - Deux systemes triphasés connectés entre enx divisent les charges y compris les charges monophasées of courts-circuits suivant les formules indiquées dans le premier article paru dans cette Revue.

Pour illustrer ces théorèmes, considérons le cas de 3 transformaleurs monophasés monlés en triangle-éloile et placons une charge monophasée ou un court-circuit entre $N$ et $B$ sur le secondaire; la répartition du courant se fera, par exemple, selon la figure 2 ci-jointe.

Considérons maintenant les transformateurs déjà étudiés du poste de Jeamne-Rose :

$1^{\text {er }}$ cas. - Charge ou court-circuit monophasé sur le primaire (en étoile), nous supposons que le secondaire (côté 15.000 volts) n'est pas relié à une station productrice d'énergie.

Le secondaire (én étoile) (figure 3) ne participe naturellement soit un courant de court-circuit de :

$$
\frac{100}{31,16}=3,2 \text { fois le courant normal. }
$$

I a répartition des courants s'effectue suivant la figure 3 (sur cette figure les rapports de transformation sont supposés égaux à 1).

$2^{e}$ cas. - Charge ou court-circuit monophasé (conducteurneutre) sur le secondaire en étoile

Nous avons vu que l'impédance normale triphasée entre secondaire et tertiaire est : $Z_{\text {st }}=16,6 \%$ (en négligeant les parties ohmiques); l'impédance en jeu entre la station génératrice $\mathrm{C}$ et le circuit $\mathrm{X}$ où la charge est appliquée est donc pour une charge monophasée égale au $\frac{1}{3}$ de la charge normale triphasér :

$$
Z_{\mathrm{cx}}=\frac{2}{3} Z_{\mathrm{ps}}+\frac{1}{3} z_{\mathrm{st}}+\frac{2}{3} z_{\mathrm{c}}
$$

or ici: $Z_{\mathrm{c}}=40,5 \%$ donc:

$$
Z_{\mathrm{cx}}=\frac{2}{3} 11, \overline{0}+\frac{1}{3} 10,6+\frac{2}{3} 40, \bar{x}=40,21 \%
$$
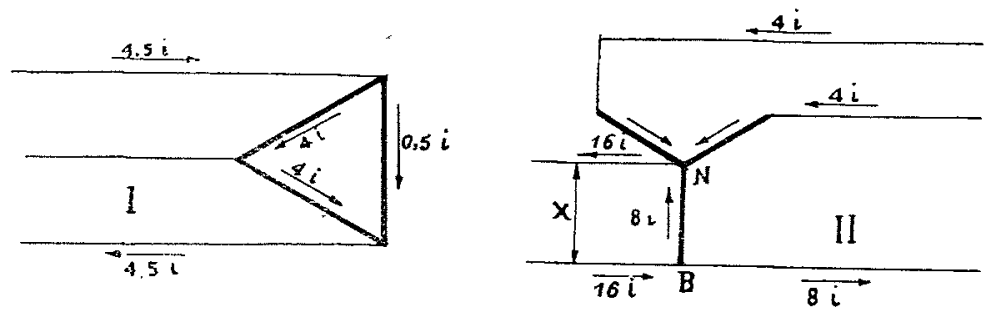

Fig. 2

Il en résulte que lors d'un court-cireuit en $X$ le courant qui y circulera sera :

$$
\frac{100}{40,21}=2,49 \text { fois le courant normal soit . }
$$
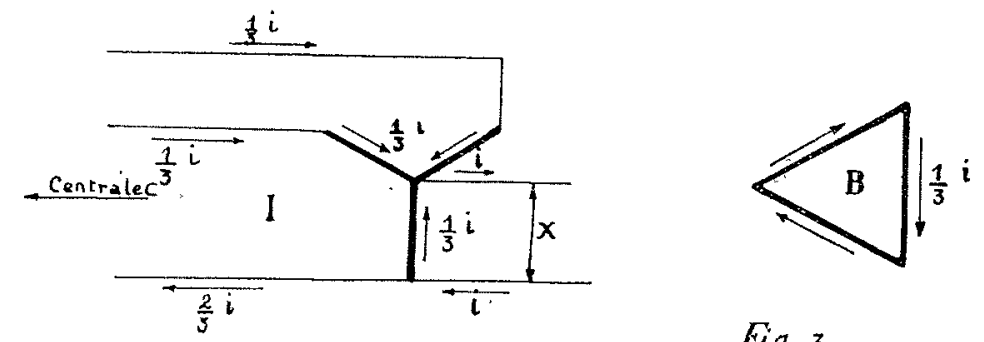

Fig. 3 pas au phénomène. Nous retombons donc dans le système simple d'un groupe de transformateurs à 2 enroulements monté en triangle-étoile. L'impédance entre le primaire (étoile) et le tertiaire (triangle) pour la charge triphasée symétrique normale est de $8,85 \%$ (voir article précédent). L'impédance effective entre le système $\mathrm{C}$ et le circuit $\mathrm{X}$ pour une charge monophaséc dans le circuit, de 3.000 KVA soit égale au tiers de la charge triphasée normale sera de :

$$
\mathrm{Z}_{\mathrm{cx}}=\frac{1}{3} 8,85+\frac{2}{3} 4,87=4,20 \%
$$

(1,87 etant la chute inductive du primaire C); pour un contcircuit l'impédance du primaire a considérer serait non pas 1,87 mais $42,37 \%$, ce qui nous donnera :

$$
z_{\mathrm{cx}}=2,96+28,2=31,16 \%
$$

$$
2,49 \times \frac{3.000 \times 1.000}{459}=287 \text { Ampères }
$$

et les KVA de court-circuit seront : $7.465 \mathrm{KVA}$.

Si nous voulons calculer le courant dans chaque circuit, en se reportant à la figure 4 nous voyons que dans le tertiaire (triangle) il circulera un courant de :

$\frac{287}{3}=95.60$ ampères reporté à $45.000 \mathrm{~V}$. soit 453 ampères à $5.500 \mathrm{~V}$.

Les 2 phases du primaire $a, b$ seront parcournes par un courant : $453 \times \frac{5.500}{67.500}=36,8$ amperes, et la $3^{\mathrm{e}}$ phase par un courant de : 73,6 ampères. 
$3^{\mathrm{e}}$ cas. - Le secondaire $(45.000 \mathrm{~V}$.) est aussi réuni à un réseau comportant une station centrale thermique (figure 5 ).

Supposons qu'un court-circuit se produise entre phase et neutre sur le secondaire A. L'impédance de chacun des deux circuits $\mathrm{C}$ et $\mathrm{A}$ par rapport au circuit $\mathrm{X}$ sera :
Remarque. - Les transformateurs étudiés pourront avoir le neutre du primaire $\mathrm{C}$ et du secondaire $\Lambda$ mis directement à la terre, soit simultanément, soit séparément.

Si dans ces conditions le neutre au départ de la Centrale $\mathrm{C}$ n'est pas à la terre et si une mise à la terre se produit, le con-
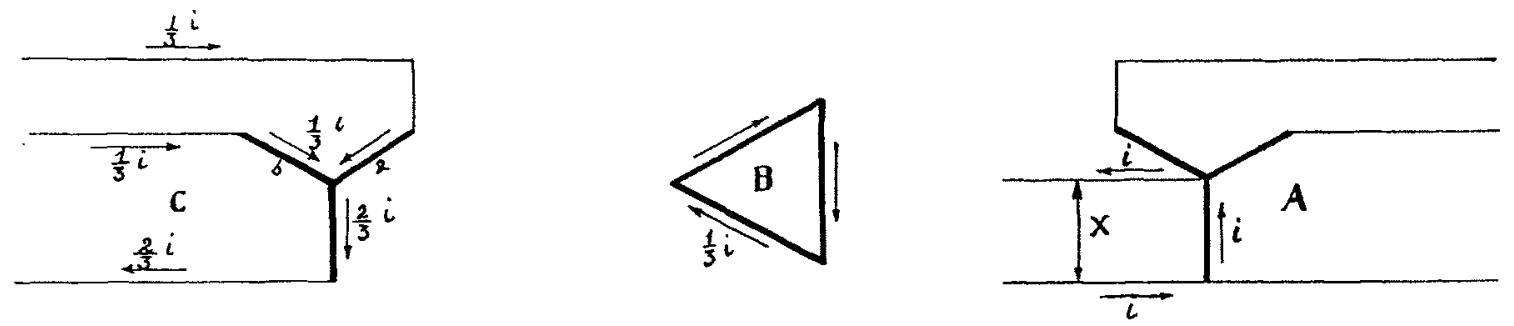

Fig.4

$$
\begin{gathered}
Z_{\mathrm{cx}}=\frac{2}{3} \mathrm{Z}_{\mathrm{c}}+\frac{2}{3} \mathrm{Z}_{\mathrm{ca}}+\frac{1}{3} \mathrm{Z}_{\mathrm{ab}}=\frac{2}{3} 40,5+\frac{2}{3} 11,5+\frac{1}{3} 16,6 \\
\ldots=40,19 \% \\
\mathrm{Z}_{\mathrm{ax}}=\frac{2}{3} \mathrm{Z}_{\mathrm{a}}+\frac{1}{3} \mathrm{Z}_{\mathrm{ab}}=\frac{2}{3} 20+\frac{1}{3} 16,6=18,86 \% \\
\mathrm{Z}_{\mathrm{ca}}=\frac{2}{3}\left(\mathrm{Z}_{\mathrm{c}}+\mathrm{Z}_{\mathrm{a}}+\mathrm{Z}_{\mathrm{ca}}\right)=48 \%
\end{gathered}
$$

rant de passage sera largement influencé par la réactance entre les deux enroulements principaux et le troisième enroulement. des transformateurs.

Si nous supposons que les rapports de transformation sont égaux à 1 (c'est-à-dire toutes les tensions et tous les courants ramenés à la haute tension), le courant circulant dans le tertiaire est toujours le $\frac{1}{3}$ du courant de terre sur le secondaire car, c'cst la seule répartition de courant qui équilibre les amperes-tours primaires et secondaires dans chaque phase.

$$
\begin{aligned}
& Z_{\mathrm{c}^{*}}=\frac{Z_{\mathrm{ca}}+Z_{\mathrm{cx}}-Z_{\mathrm{ax}}}{2}=34,66 \% \\
& \mathrm{Z}_{\mathrm{a}^{\prime}}=\frac{\mathrm{Z}_{\mathrm{ax}}+\mathrm{Z}_{\mathrm{ca}}-\mathrm{Z}_{\mathrm{cx}}}{2}=13,34 \% \\
& \mathrm{Z}_{\mathrm{x}^{\prime}}=\frac{\mathrm{Z}_{\mathrm{cx}}+\mathrm{Z}_{\mathrm{ax}}-\mathrm{Z}_{\mathrm{ca}}}{2}=5,33 \%
\end{aligned}
$$

Si le transformateur étudić était réuni à des réseaux de grande capacité et qu'il y ait une faible réactance de génératrices et de lignes de transmission, l'influence des impédances dans le transformateur lui-même serait prédominante; si, au contraire, comme dans le cas actuel, les centrales sont éloignées et les
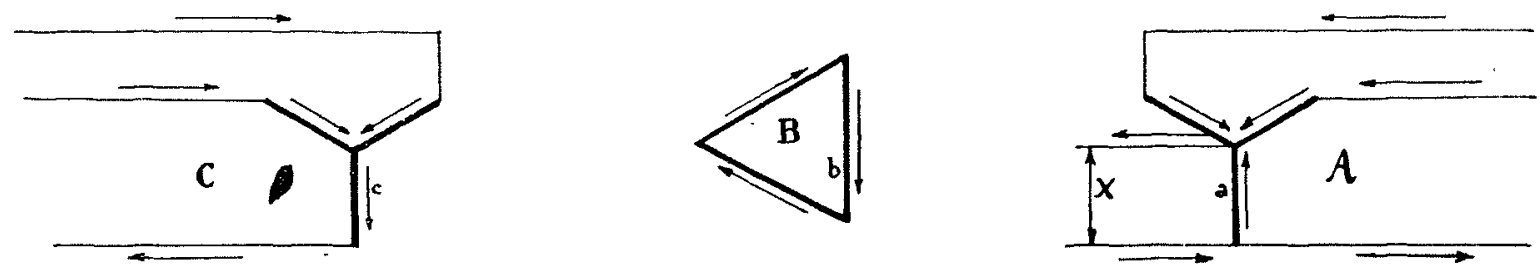

Fig. 5

Pour le court-circuit considéré l'impédance totale du réseau C, A et du transformateur sera :

$$
\mathrm{Z}=\frac{\mathrm{Z}_{\mathrm{ax}} \mathrm{Z}_{\mathrm{cx}}-\mathrm{Z}_{\mathrm{x}}^{2}}{\mathrm{Z}_{\mathrm{ca}}}=15,18 \%
$$

et les KVA alimentant le court-circuit seront :

$$
3.000 \times \frac{100}{15,18}=19.800 \mathrm{KVA} \text { dont une partie : }
$$

$19.800 \times \frac{Z_{\mathbf{c}^{\prime}}}{Z_{\text {ca }}}=14.380 \mathrm{KVA}$ sera fournie par le réseau A, et $5.420 \mathrm{KVA}$ par le réseau $\mathrm{C}$; le courant total sera

$\frac{100}{15,18}$ soit 6,6 fois le courant normal, dont 4,77 fois proviennent du réseau $\mathrm{A}$ et 1,83 du réseau ${ }^{-} \mathrm{C}$. alternateurs ont de fortes chutes de tension, les impédances du transformateur ont une influence moins marquée.

Nous avons toujours supposé que la tension se maintenait constante lors d'un court-circuit, alors que pratiquement elle ne le sera pas tout à fait et la valeur du courant de court-circuit sera plus faible que celle calculée plus haut.

Les courts-circuits que nous avons étudiés sont des courtscircuits permanents, c'est-à-dire ont pris la valeur qu'ils atteignent au bout d'un certain nombre de sccondes; la valeur instantanée serait d'environ 3 fois la valeur permanente, mais les disjoncteurs du réseau ne déclanchant jamais avant 3 secondes, ils n'auront à couper que la valeur permanente de courtcircuit. 